\title{
A Systematic Review of the Influence of Continuous Positive Airway Pressure on Fetal and Newborn Animal Models: Suggestions to Improve Neonatal Respiratory Care
}

\author{
Andre George Gie ${ }^{a}$ Talia Rose Hubble ${ }^{b}$ Yannick Regin ${ }^{a}$ Thomas Salaets ${ }^{a}$ \\ Monica Zamora ${ }^{c}$ Jan Deprest ${ }^{a, d}$ Jaan Toelen ${ }^{a}$ \\ ${ }^{a}$ Department of Development and Regeneration, KU Leuven, Leuven, Belgium; ${ }^{b}$ Medical Science Division, University \\ of Oxford, Oxford, UK; ' ${ }^{B}$ CNatal, Fetal Medicine Research Center, Hospital Clinic and Hospital Sant Joan de Déu, \\ University of Barcelona, Barcelona, Spain; 'Institute for Woman's Health, University College London Hospital, \\ London, UK
}

\section{Keywords}

Continuous positive airway pressure - Ventilation ·

Respiratory function - Bronchopulmonary dysplasia

\begin{abstract}
Introduction: Prematurely born infants regularly develop respiratory distress syndrome and require assisted ventilation. Ventilation may injure the premature lung and increase the risk of bronchopulmonary dysplasia. Continuous positive airway pressure (CPAP), a form of noninvasive ventilation, is commonly used in modern neonatology. Limited clinical data are available on the acute and long-term effect of neonatal exposure to CPAP on the lung. Given the restricted clinical data, newborn animal models have been used to study the influence of CPAP on lung structure and function. The findings of animal studies can guide neonatal care and improve the use of CPAP. Methods: A systematic review of electronic databases (Medline, Embase, and Cinahl) was performed using the medical subject heading terms, "CPAP" or "continuous positive airway pressure" and "animals" and "newborn." Abstracts were screened for inclusion using predetermined eligibility criteria. Results: In total, 235 abstracts were identified and screened for inclusion. Of these, 21 pa-
\end{abstract}

pers were included. Large $(N=18)$ and small $(N=3)$ animal models investigated the effects of CPAP. Pulmonary outcomes included gas exchange, lung structure and function, surfactant metabolism, lung inflammation and injury, and the effect of intrapulmonary therapy. Compared to mechanical ventilation, CPAP improves lung function, evokes less lung injury, and does not disrupt alveolar development. Surfactant administration combined with CPAP further improves respiratory outcomes. Of concern are findings that CPAP may increase airway reactivity. Discussion/Conclusion: CPAP offers numerous advantages over mechanical ventilation for the immature lung. The combination of CPAP and exogenous surfactant administration offers further pulmonary benefit.

() 2020 S. Karger AG, Basel

\section{Introduction}

Preterm infants frequently develop respiratory distress syndrome (RDS) requiring assisted ventilation to survive. While necessary in the acute setting, ventilation may injure the newborn lung and increase the risk of developing bronchopulmonary dysplasia (BPD) [1]. karger@karger.com

(C) 2020 S. Karger AG, Basel

www.karger.com/neo

Karger $\stackrel{2}{*}$
Jaan Toelen

Department of Development and Regeneration

KU Leuven, Herestraat 49

BE-3000 Leuven (Belgium)

jaan.toelen@uzleuven.be 
Table 1. Systematic review of the influence of CPAP on newborn and fetal animal models

\begin{tabular}{ll}
\hline Inclusion criteria & Exclusion criteria \\
\hline In vivo animal models & Ex vivo lung models \\
\hline Animal study & Human study \\
\hline Fetal and newborn & Juvenile and adult \\
\hline CPAP as the primary intervention & $\begin{array}{l}\text { Mechanical ventilation including intermittent positive-pressure ventilation, } \\
\text { high frequency oscillation ventilation, jet insufflation, and sustained inflation }\end{array}$ \\
\hline No surgical intervention (excluding airway insertion) & Surgical intervention to respiratory system, for example, CDH creation \\
\hline Lung function and/or structural readouts & No lung structure or functional readouts \\
\hline Novel research paper & Review paper \\
\hline
\end{tabular}

Suggestions to improve neonatal respiratory care, study eligibility, and inclusion and exclusion criteria are presented in the table. CPAP, continuous positive airway pressure.

Continuous positive airway pressure (CPAP) is a commonly used form of assisted ventilation and is recommended as the initial respiratory support for spontaneously breathing infants [2]. CPAP applies a continuous distending pressure to the lung, limiting atelectasis found associated with surfactant deficiency and stabilizing the compliant chest wall of extremely preterm infants. CPAP avoids large tidal volumes, and appears to reduce lung injury and the incidence of BPD [3-6].

Neonatal lung injury and BPD have lifelong consequences. Infants fail to reach their full respiratory potential, exhibit worse lung function than their peers throughout life and are at risk to develop early chronic obstructive airway disease [7-9]. Additionally, BPD is independently associated with poorer neurological outcomes [10-12]. Strategies to minimize lung injury include limiting oxygen exposure, volume-protective ventilation, and noninvasive ventilation such as CPAP. However, CPAP may not be free of long-term consequence and has been associated with an increased duration of oxygen therapy and airway obstruction later in life [13].

While CPAP has been enthusiastically adopted into clinical practice, its effect on the function and structure of the developing lung beyond the initial resuscitation period remains unclear [14]. Clinical studies have demonstrated CPAP to be effective in the acute management of RDS following preterm birth and have described the influence of neonatal respiratory management on the incidence of BPD and pulmonary function in childhood $[13,15]$. Human data are limited by the difficulty of performing lung function tests in newborn infants and the lack of access to representative lung tissue. Therefore, animal studies have been employed to examine the consequences of CPAP on the immature lung. This paper reviews the effect of CPAP compared to other forms of ventilation, on the structure and function of the developing lung in fetal and newborn animal models, and suggests improvements to neonatal respiratory care based on the findings.

\section{Methods}

We systematically searched Medline, Embase, and Cinahl databases using medical subject heading terms, "CPAP" or "continuous positive airway pressure" and "animals" and "newborn." The abstracts of the papers identified in the electronic search were screened for inclusion according to predetermined eligibility criteria (Table 1). The references of included papers were manually screened to identify additional papers that met the study eligibility criteria. The search was updated on March 31, 2020.

\section{Study Eligibility Criteria}

In vivo fetal or newborn animal studies were included (Table 1). We defined the newborn period as the first 14 days postdelivery. We defined CPAP as a method of respiratory support in animals with spontaneous respiratory efforts, which provided a continuous distending pressure to the airways without intermittent increases in peak pressure. We included CPAP applied with both invasive (endotracheal intubation) and noninvasive (maskface or nasal interface) methods used to apply the distending pressure. Specifically we included ventilator-driven CPAP, conventional CPAP apparatus, and bubble CPAP systems. Studies that used mechanical ventilation for stabilization following birth and converted respiratory management to CPAP to compare CPAP to continued mechanical ventilation were included. We did not include intermittent positive pressure ventilation studies that examined the effect of positive-end expiratory pressure in mechanical 
Table 2. Systematic review of the influence of CPAP on newborn and fetal animal models

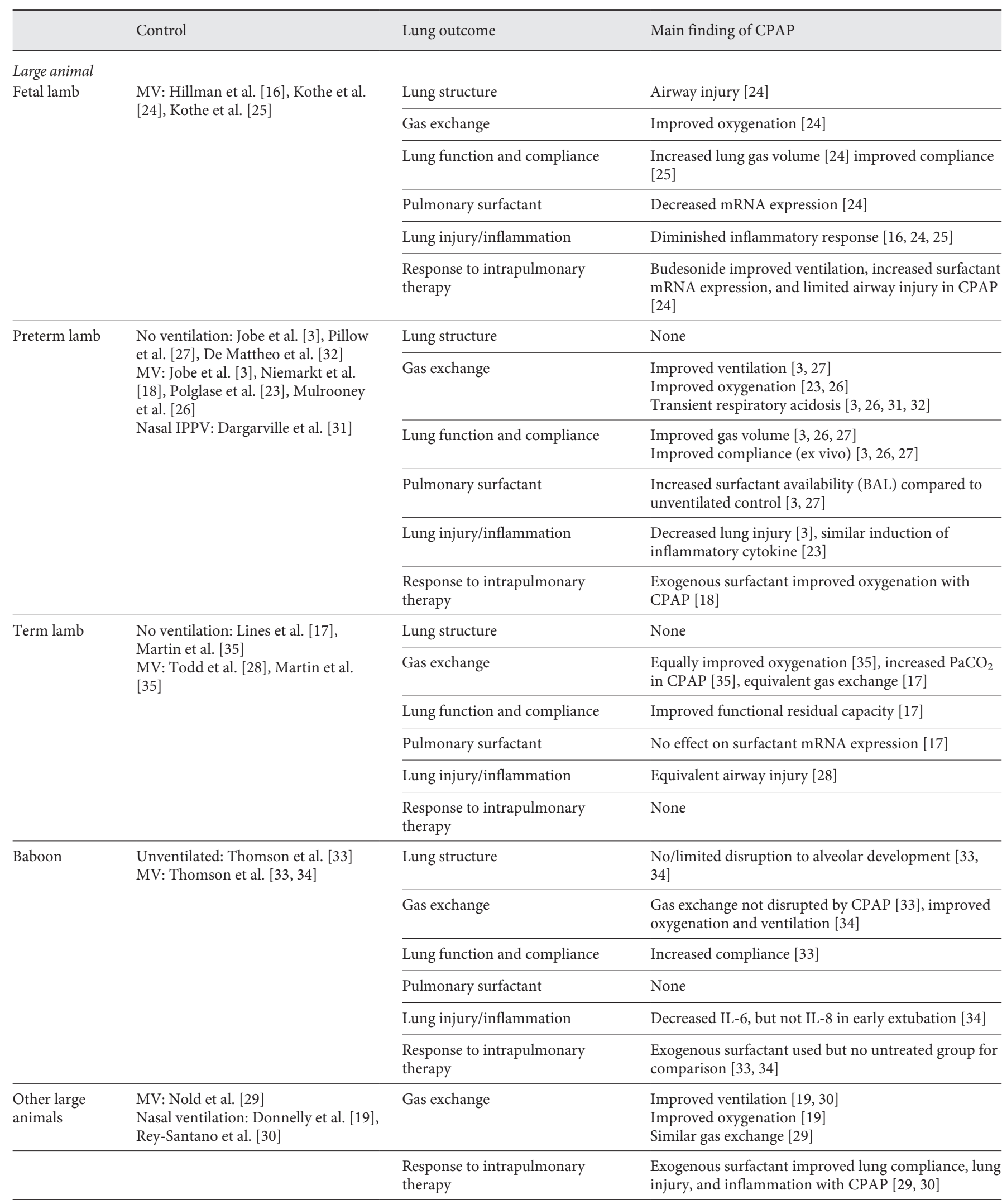


Table 2 (continued)

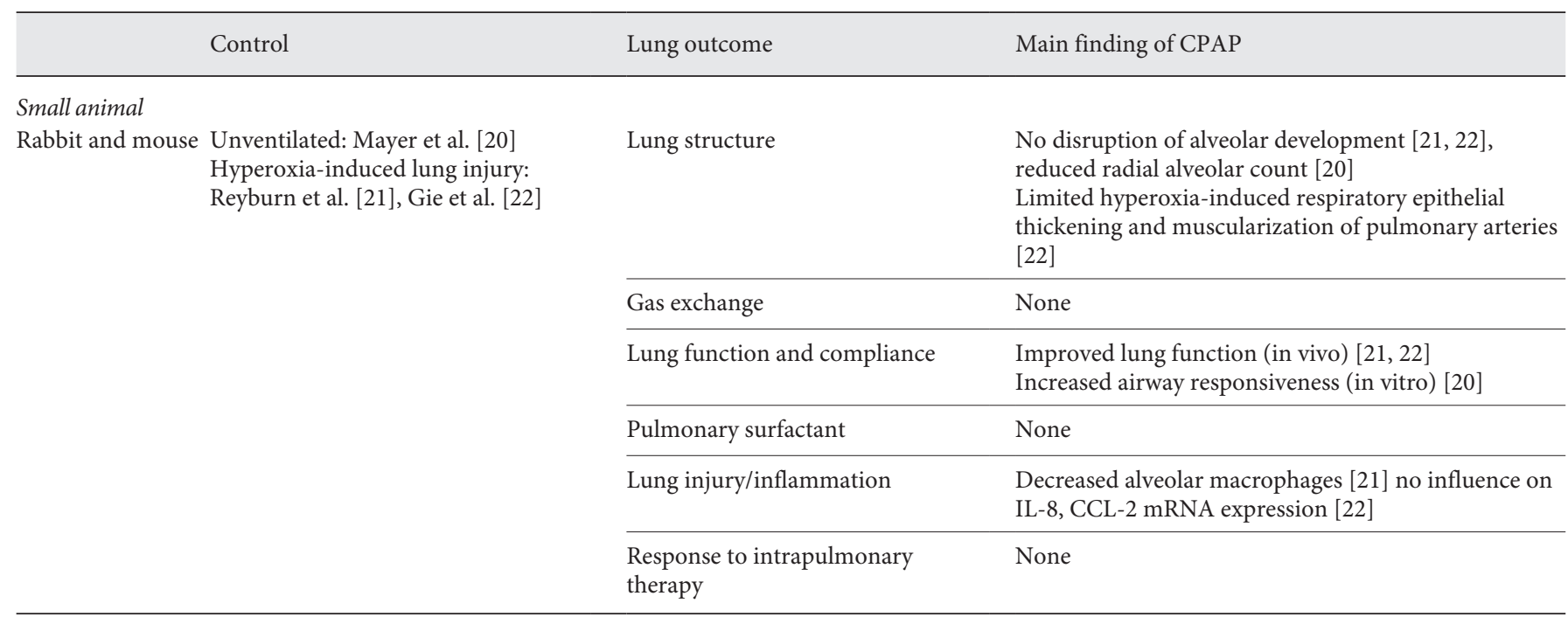

Suggestions to improve neonatal respiratory care and the main findings of the effect of CPAP on pulmonary outcomes in fetal and newborn animal models are presented in the table. CPAP, continuous positive airway pressure; MV, mechanical ventilation; mRNA, messenger RNA; BAL, bronchoalveolar lavage; $\mathrm{PaCO}_{2}$, partial pressure of carbon dioxide in arterial blood; IL-6, interleukin 6; IL-8, interleukin 8; CCL-2, chemokine ligand 2.

ventilation, high-frequency oscillation ventilation techniques, and sustained inflation maneuvers. Studies that did not examine pulmonary outcomes were excluded.

\section{Quality Appraisal}

We assessed the risk of bias and study quality by using a checklist developed by the group of Collaborative Approach to MetaAnalysis and Review of Animal Data from Experimental Studies (CAMARADES) (see online suppl. Table 1; for all online suppl. material, see www.karger.com/doi/10.1159/000511086).

\section{Data Collection}

AG and TH performed literature search, screened the papers and extracted the data.

\section{Results}

A total of 236 abstracts were identified and screened for inclusion. Of these, 99 (42\%) were excluded (duplicates $[n=29,29 \%]$, review articles $[n=46,46 \%]$, conference abstracts, case reports or feasibility studies $[n=24$, $24 \%])$. Of the remaining 136 papers, a further $116(86 \%)$ were not included because CPAP was not the primary intervention $(n=52)$, human studies $(n=22)$, lung outcomes not assessed $(n=23)$, or the study was undertaken outside the newborn period $(n=19)$. Thus, a total of 21 papers are included in this review.
Various animal models have been employed to examine the effect of CPAP on the lung. Large animal models including fetal, preterm, and term lambs, piglets, and calves have been used to investigate the acute effect of minutes to hours of CPAP on the lung, while preterm baboons have been employed to investigate the effects of CPAP beyond the first days of life. Small animal models, preterm rabbits, and term mice, have examined the effect of multiple days of intermittent CPAP. These models have reported various outcomes including lung structure, gas exchange, lung function and compliance, pulmonary surfactant, lung injury and inflammation, and the effect of intrapulmonary therapy. The main findings of the studies are summarized in Table 2. An overview of the CPAP models used (duration and pressure of CPAP) and pulmonary outcomes of the studies is available in online suppl. Table 2 .

\section{Quality Appraisal}

Common strengths of the studies include publication in peer-reviewed journals, lack of animals with comorbid disease, and compliance with animal welfare regulatory requirements (online suppl. Table 1). The majority randomized animals to intervention groups $[3,16-30]$ and reported temperature control [3, 17-22, 27-34]. Weaknesses include few studies which blinded all outcome measures $[3,16,24]$, or avoided anesthesia with intrinsic 
effect on the lung [18, 27, 31-34]. A single study reported sample size calculation, while no studies concealed intervention allocation [22]. A number of studies reported financial support but did not clearly report whether a conflict of interest was present $[3,16,17,20,21,23,28,29$, $34,35]$.

\section{CPAP Delivery}

A variety of CPAP delivery systems, duration, and airway pressure were employed by the studies (online suppl. Table 2). Endotracheal intubation was performed by a large proportion of the animal studies $[3,16,17,23-30$, 33-35]. Following stabilization, extubation to noninvasive CPAP delivery was performed in the baboon and pig models $[29,30,33,34]$. Both large and small animal models avoided endotracheal intubation and delivered CPAP noninvasively to more closely replicate clinical practice $[18,20-22,31,32]$ The median duration of CPAP was $3 \mathrm{~h}$ (range $10 \mathrm{~min},>9$ days). The duration of CPAP used varied including CPAP of $<1 \mathrm{~h}[16,19,24,25], 1-2 \mathrm{~h}[3]$, 2-6 h [18, 23, 26-31, 35], 6-24 h [17, 28, 32], and multiple days of CPAP $[33,34]$. Unlike the large animal models, the small animals models investigated the effect of multiple days of intermittent CPAP on the developing lung [20-22].

\section{Lung Structure}

Studies examined the structural effect of CPAP applied during the cannicular (baboon), saccular (preterm sheep, rabbit, mouse) and alveolar (term sheep, piglet, calf) phase of lung development. CPAP did not decrease alveolar surface area, increase fibrosis, or disrupt development of the alveolar wall and pulmonary microvasculature compared to unventilated age-matched controls [33]. Early extubation to CPAP attenuated ventilationinduced alveolar injury [33, 34]. Multiple days of intermittent CPAP was associated with an increase in radial alveolar count in moderate hyperoxia but did not alter alveolar structure in severe hyperoxia in the saccular phase of lung development $[21,22]$.

\section{Gas Exchange}

Large animal models examined gas exchange using blood gas analysis. Preterm CPAP-treated animals had transient respiratory acidosis which did not compromise cardiovascular stability and resolved as respiratory drive increased [3, 26, 31, 32]. Provided respiratory drive is sufficient, CPAP improves ventilation and oxygenation [3, $19,24,26,27,30,34,35]$.

Systematic Review of CPAP in Newborn Animals

\section{Lung Function and Compliance}

Large animal models examined ex vivo pressure-volume curves and functional residual capacity (FRC). These studies determined that CPAP increased lung gas volume and compliance [3, 17, 24-27, 33]. In vivo lung function was examined in small animal models using both pressure-volume maneuvers and oscillometry. CPAP improved lung mechanics as measured by oscillometry but did alter inspiratory capacity or in vivo static compliance in models of hyperoxia-induced lung injury [21, 22]. Examination of ex vivo airway reactivity found CPAP to increase the airway response to methacholine [20]. No study investigated if CPAP increased in bronchoconstriction or altered in vivo expiratory airflow.

\section{Pulmonary Surfactant}

The effect of CPAP on endogenous pulmonary surfactant was examined in the lamb. In fetal lambs, CPAP decreased mRNA expression of surfactant proteins, while CPAP had no effect on the expression of surfactant proteins in term lambs $[17,24]$. CPAP increased the amount of saturated phosphatidylcholine in the bronchoalveolar lavage fluid of preterm lambs compared to unventilated controls $[3,27]$.

\section{Lung Injury and Inflammation}

Studies examined the influence of CPAP on histological lung inflammation score $[3,29,30]$, histological airway inflammation score [28, 34], lung wet-dry ratio [27, 29], alveolar macrophage content [21], bronchoalveolar fluid cell count $[3,16,26,29,33,34]$, and inflammatory cytokine mRNA expression [3, 16, 22-26, 30, 33, 34]. Compared to mechanical ventilation (MV), CPAP invoked less inflammatory response and lung injury with decreased bronchoalveolar fluid neutrophil count [3], and unlike MV, did not induce expression of Il-1 $\beta[16$, $25]$. The influence of CPAP on IL-6 and IL- 8 mRNA expression is not clear as studies have described conflicting results $[3,23,26,33,34]$. Compared to hyperoxia-exposed unventilated controls, CPAP did not increase the number of alveolar macrophages or increase the mRNA expression of Il-8 or CCL-2 $[21,22]$. Bubble CPAP further limits lung injury compared to conventional CPAP and is not associated with an increase in alveolar protein content [27]. In contrast, if intubated, airway injury is comparable in CPAP and MV with similarly increased IL- 8 and IL-1 $\beta$ mRNA expression in the tracheal mucosa [26]. In surfactant deficient models, CPAP in the absence of surfactant replacement increased lung injury and inflammation $[29,30]$ In these models, surfactant replace- 
ment limited lung inflammation and injury while improving lung compliance and oxygenation $[18,29,30]$.

\section{Intrapulmonary Therapy}

Exogenous pulmonary surfactant supplementation was used in several studies and improved gas exchange in both CPAP and MV $[18,29,30]$. A single study investigated intrapulmonary budesonide combined with CPAP. While combined budesonide-CPAP therapy did not reduce lung inflammation, it reduced airway injury and increased surfactant mRNA expression [24]. A single study examined whether combined intratracheal and intravenous epidermal growth factor receptor inhibition would limit airway injury and lung inflammation, finding no benefit in either mechanical ventilation or CPAP [25].

\section{Discussion}

Despite CPAP being widely used for respiratory support in preterm infants, surprisingly little is known of its functional and structural effect(s) on the immature lung. Due to the difficulty of examining lung function and structure in ill neonates, animal models have been employed to investigate the effect of CPAP.

The quality of the included animal studies was reviewed using a system specifically designed for animal models (CAMARADES). The interpretation of these results can be difficult, as the total score does not necessarily indicate the study quality, and each category may not carry equal weight. Nonetheless they provide a framework to identify strengths and weaknesses of animal studies. In reviewing these papers, we identified several common strengths and weaknesses. Limitations such as the lack of blinding of treatment allocation, which would be difficult while managing animals with differing respiratory management, and blood gas analysis (an automated process) are understandable though they limit the internal validity of the studies. The quality of several studies could be improved by the inclusion of a power calculation and a specific statement regarding potential conflicts of interest in addition to declaring sources of financial support.

The models employed to examine the influence of CPAP on the developing lung can be broadly categorized into large (baboon, sheep, pig, calf) and small animals (rabbit, mouse). Each of these models offers certain advantages and disadvantages. The lung development of large animal models closely resembles human lung development and has the greatest translational value. Further- more, the lamb and baboon can be delivered prematurely, develop respiratory distress and can be managed with interventions which replicate that of modern neonatal care. Large animal models have the advantage of large blood vessels and blood volume, which allows for repeated blood gas analysis and the detailed investigation of the influence of CPAP on pulmonary gas exchange. Additionally, studies have also been performed on fetal animals, which allow the isolation of the effects of ventilation and oxygen exposure. In vivo testing of lung compliance, inspiratory capacity, and lung mechanics is limited in the large animal models. While it possible to examine lung mechanics in noninvasively ventilated large animals with oscillometry, this technique has only been performed in a single large animal study of the effect of CPAP [27, 36, 37]. Thus, the effect of CPAP on lung function in large animals, beyond that of gas exchanges, remains unclear and requires further investigation. The significant cost and intensity of care required for long-term large animal experiments limit the investigation of long-term CPAP studies. Baboon studies have great translational value, as a result of lung development similar to the human, and the development of respiratory distress and BPD following preterm birth, but are rarely performed due to ethical concerns.

Small animal models have the advantage of relatively low cost and short gestational times, and allow for in vivo lung function testing. A wide range of molecular tests is available to study pathophysiological processes in mice, while rabbits can be born prematurely prior to the alveolar phase of development allowing for a better approximation of the human clinical scenario. A major limiting factor of the small animal models is the use of intermittent as opposed to continuous CPAP as used in clinical neonatology. The mouse model is further limited by the inability to survive preterm birth. While term mice are delivered in the saccular phase of lung development, the lungs are functionally mature and may not mimic the lung of the preterm human infant. Rabbit models have limited commercial molecular and protein tests available and are unable to give insight into the mechanism by which interventions exert their influence. It is interesting to note that the rat model - even though it is often used as a neonatal BPD model - was not yet used to study CPAP.

To further complicate the comparison between the animal models, different methods of CPAP delivery including conventional and bubble CPAP have been used with only a single study directly comparing the 2 methods. The use of endotracheal intubation makes certain results, 
such as airway injury, difficult to interpret. Intubation may induce airway injury and does not reflect the clinical practice of noninvasive CPAP. Progress in the use of noninvasive ventilation techniques in large animal models may allow future experiments to compare noninvasive to invasive CPAP and MV [19, 31, 33, 34, 38]. Further handicapping the clinical translation of animal studies data is the short duration of CPAP used in several of the large animal models and the intermittent use of CPAP by the small animal models. While not reflecting clinical practice, these studies demonstrate that limited or intermittent exposure to CPAP may have multiple effects on the immature lung; however, it remains unclear if these effects influence survival or long-term lung structure and function. Future animal studies should investigate the influence of continuous, noninvasive CPAP on lung structure and function and whether CPAP-induced changes persist beyond the neonatal period.

A variety of lung outcomes have been reported. For the purpose of this review, the outcomes will be discussed in the following categories: lung structure, respiratory physiology, lung compliance and function, pulmonary surfactant, lung inflammation and injury, and intrapulmonary therapy.

\section{Lung Structure}

Due to the difficulties of extended CPAP application in animal models, relatively few studies have addressed the long-term effect of CPAP on lung structure. The majority of studies examining the acute affect of CPAP can examine indicators of lung injury but are unable to examine alveolar development. Of the studies, the preterm baboon model provides the highest quality evidence of the long-term effect of CPAP on lung structure and alveolar development. The model combined birth in the cannicular phase of lung development, and detailed examination of the alveolar structure and care similar to that of modern neonatal intensive care. Encouragingly, multiple days of CPAP exposure, following initial mechanical ventilation, did not stunt alveolar development $[33,34]$. Supporting evidence is provided by small animal studies demonstrating that multiple days of intermittent CPAP exposure during the saccular phase of lung development did contribute to alveolar injury. Additionally, CPAP may benefit lung development and limit airway injury in moderate hyperoxia [21]. These findings are encouraging for the use of CPAP in preterm infants born prior to the alveolar phase of lung development. CPAP may not contribute to alveolar injury and the disruption of alveolar development, and key factors in the development of BPD following preterm birth. However, further animal studies are imperative to investigate the influence of CPAP on long-term lung structure and alveolar development.

\section{Gas Exchange}

Improved gas exchange is the primary reason for the initiation of respiratory support following preterm birth. Studies examining CPAP aim to test the hypothesis that noninvasive ventilation can improve gas exchange while avoiding the complications of invasive ventilation. Animal studies demonstrate that oxygenation can be improved or maintained by CPAP, while hypercarbia may take some time to resolve [3, 19, 26, 30-33]. When CPAP is unable to maintain oxygenation, MV or nasal oxygen insufflation improves gas exchange $[19,35]$. Transient respiratory acidosis did not precipitate cardiovascular instability and resolved as respiratory drive increased [26, 31]. Should respiratory acidosis be concerning, bubble CPAP appears to be advantageous to conventional CPAP [27]. Additionally, bubble CPAP may improve oxygenation compared to conventional CPAP; however, only a single study compared the 2 modalities [27]. A single study found gas exchange to be influenced by gender, with preterm male lambs having higher oxygen requirements and poorer ventilation than females [32]. Should initial endotracheal intubation be required, a single study provides evidence favoring early extubation to CPAP. Early extubation improved respiratory drive and function compared to delayed extubation and prolonged MV [34].

Animal studies support the use of CPAP as respiratory therapy for newborn infants. CPAP improves oxygenation and ventilation, limiting the need for endotracheal intubation. Transient hypercarbia should not discourage the use of CPAP. Clinical studies have demonstrated moderate hypercarbia is not associated with poorer longterm outcome [39]. Thus, animal models support the clinical practice that the use of CPAP is justified, provided the infant is adequately oxygenated and remains cardiovascularly stable. Similarly, the practice of weaning from MV to CPAP is supported in animal models [2].

\section{Lung Function and Compliance}

Animal studies have examined ex vivo lung gas volume and compliance, and in vivo lung function testing. CPAP increases the FRC. The increase in FRC improved gas exchange in bubble CPAP but not conventional CPAP $[17,27]$. This may be related to limitations of the term model used in the conventional CPAP model, as the term animals are unlikely to have RDS or atelectasis. A further limitation of the FRC findings is the short duration of the 
studies, as derecruitment and atelectasis may occur over time. Clinical studies have demonstrated that extended CPAP increases FRC in infants [40]. Yet an increased FRC may not be beneficial to all infants. In infants with established BPD, the increase in FRC following CPAP did not improve gas exchange [41]. In addition to FRC, CPAP increased ex vivo lung compliance $[3,26]$. Further evidence of benefit is provided by early extubation to CPAP improving compliance compared to continued MV [34].

Similar to human studies, in vivo lung function data in animal models are scarce. Small animal studies have shown CPAP to improve in vivo lung function in hyperoxia lung injury models $[21,22]$. However, it remains unclear whether these findings translate into improved long-term lung function.

The lung function tests performed in the included small animal studies relied on the FlexiVent system, which allows for in vivo pressure-volume maneuvers and oscillometry. However, unlike full body plethysmography, the FlexiVent system is limited by the requirement for anesthesia and tracheostomy and did not allow for repeated lung function evaluation in the included studies. Data on airway responsiveness and expiratory airflow are limited despite abnormal expiratory airflow being a common clinical finding following BPD. Future studies, both human and animal, should examine whether atelectasis occurs with prolonged CPAP use, in vivo lung function and the long-term effect of CPAP on airway responsiveness and expiratory airflow.

\section{Pulmonary Surfactant}

Ventilation may alter surfactant production, metabolism, and availability. Preterm lambs studies demonstrate CPAP increases surfactant availability in bronchoalveolar lavage fluid, possibly explaining the increase in ex vivo lung compliance $[3,27]$. It remains unclear whether an increase in surfactant availability following CPAP has lasting physiological benefit and exogenous surfactant administration remains a key therapy in the management of RDS. Less-invasive surfactant replacement administration was found to have similar physiological benefits to endotracheal tube surfactant administration, despite less surfactant deposition in the lung [18]. Minimally invasive surfactant techniques that avoid the need for manipulation of the airway may offer similar advantages to lessinvasive surfactant replacement administration. Nebulization of surfactant to allow delivery to the peripheral lung is an appealing treatment option and has been shown to improve oxygenation and lung function of animals on CPAP [42]. Animal models provide evidence that sup- port the use of CPAP combined with exogenous surfactant to improve oxygenation and limit lung injury $[18,29$, 30]. Animal models can continue to contribute to improve surfactant supplementation by investigating techniques to improve surfactant distribution to limit the invasiveness of surfactant delivery.

\section{Lung Injury and Inflammation}

Ventilation-induced lung injury is a potentially modifiable risk factor of neonatal lung injury and BPD. CPAP has been proposed as an alternative ventilation strategy to limit acute lung injury compared to conventional mechanical ventilation. Clinical studies are scarce due to the difficulty of objectively quantifying lung injury beyond blood gas analysis and lung imaging. Encouragingly, in animal models, histological indicators of lung injury and markers of lung inflammation were lower in CPAP than MV and in early extubation from MV to CPAP $[3,16,24$, 34]. However, the effect of CPAP on lung inflammation and injury is not clear as animal studies have examined many variations of CPAP in multiple animal models, making the comparison of study findings difficult, nor is it clear whether decreased lung injury in the neonatal period directly decreases the incidence of $\mathrm{BPD}$ and improves long-term lung function following extremely preterm birth. The limited data on the effect of CPAP on the airway suggest that airway injury is largely related to intubation and physical factors such as gas humidity [24, 28]. Overall, animal studies indicate CPAP limits both lung and airway injury provided intubation is avoided or limited and the delivered gas is adequately humidified. These findings support the use of CPAP as the initial mode of respiratory support in clinical practice. Future studies should investigate whether limiting early lung and airway injury has long-term benefits.

\section{Intrapulmonary Medication}

The use of intrapulmonary medication to limit lung injury is an appealing strategy. A single study found that budesonide-surfactant prevents extracellular matrix- and airway-remodeling following fetal ventilation [24]. Clinical studies have demonstrated that the combination of budesonide and surfactant improves neonatal outcomes in mechanically ventilated preterm infants $[43,44]$. However, to date no study has addressed the combination of prolonged CPAP and budesonide-surfactant. Additional intratracheal therapeutics including the modulation of the signaling of pathways associated with ventilation-induced injury, such as inhibition of epidermal growth factor receptor signaling, have not been successful in pre-
12

Neonatology 2021;118:5-14 DOI: $10.1159 / 000511086$
Gie/Hubble/Regin/Salaets/Zamora/ Deprest/Toelen 
venting acute ventilation-induced lung injury [25]. Apart from exogenous surfactant supplementation, no intrapulmonary therapy for neonatal lung disease has sufficient data to clearly guide therapy. The intratracheal administration of budesonide shows promise; however, the data on its effect on the lung remains limited. Clinicians should carefully consider the benefit of intratracheal budesonide-surfactant, in preterm infants at high risk of lung injury. Animal studies examining intrapulmonary therapies are required to improve intrapulmonary drug delivery techniques and identify potential interventions to limit neonatal lung injury.

In summary, numerous advantageous and deleterious effects of CPAP have been described in animal models. The use of these animal data to guide clinical practice is limited by the fact that CPAP has been applied by invasive and noninvasive methods for varying periods of time and pressure. Similarly, different outcomes have been reported. Given the difficulty of obtaining detailed data from sick neonates receiving CPAP, animal models will continue to play an important role in optimizing neonatal care.

\section{Conclusion}

CPAP influences multiple aspects of the developing lung. Animal studies provide evidence to support the use of CPAP over mechanical ventilation, early extubation to CPAP, and surfactant supplementation. CPAP does not appear to disrupt alveolar development, but it is not without risk and potentially increases airway reactivity. Future studies should further examine the functional and structural effects of CPAP on the newborn lung to guide neonatal ventilation strategies.

\section{Statement of Ethics}

None of the authors have any ethical conflicts to disclose. This study was exempt from ethical approval as it reviewed published literature and did not perform any human or animal experiments.

\section{Conflict of Interest Statement}

The authors have no conflicts of interest to declare.

\section{Funding Sources}

This research was supported by a C2 grant from KU Leuven (C24/18/101) and a research grant from the Research Foundation - Flanders (FWO G0C4419 N). A.G. is supported by the Erasmus + Program of the European Commission (2013-0040). Y.R. is holder of an FWO-SB fellowship (Research Foundation - Flanders, $1 \mathrm{~S} 71619$ N). J.D. is partly funded by the Great Ormond Street Hospital Charity Fund. None of the funding bodies was involved in the design of the study and in the collection, analysis, and interpretation of data.

\section{Author Contributions}

A.G., T.H., J.D., and J.T. conceived and designed the study; A.G. and T.L. performed the literature search and extracted the data; A.G. and T.L. drafted the manuscript; A.G, T.H., Y.R., T.S., M.Z., and J.T. edited and revised the manuscript; and A.G., T.H., Y.R., T.S., M.Z., J.D., and J.T. approved the final version of the manuscript.

\section{References}

1 Stoll BJ, Hansen NI, Bell EF, Walsh MC, Carlo WA, Shankaran S, et al. Trends in care practices, morbidity, and mortality of extremely preterm Neonates, 1993-2012. JAMA. 2015;314(10):1039-51.

2 Sweet DG, Carnielli V, Greisen G, Hallman M, Ozek E, Te Pas A, et al. European consensus guidelines on the management of respiratory distress syndrome - 2019 update. Neonatology. 2019;115(4):432-50.

3 Jobe AH, Kramer BW, Moss TJ, Newnham JP, Ikegami M. Decreased indicators of lung injury with continuous positive expiratory pressure in preterm lambs. Pediatr Res. 2002; 52(3):387-92.

4 Wright CJ, Polin RA. Noninvasive support: does it really decrease bronchopulmonary dysplasia? Clin Perinatol. 2016;43(4):783-98.
5 Schmölzer GM, Kumar M, Pichler G, Aziz K, O’Reilly M, Cheung PY. Non-invasive versus invasive respiratory support in preterm infants at birth: systematic review and metaanalysis. BMJ. 2013 Oct 17;347:f5980.

6 Keszler M, Sant'Anna G. Mechanical ventilation and bronchopulmonary dysplasia. Clinics in perinatology. Elsevier Inc.; 2015. Vol. 42; p. 781-96.

7 Bolton CE, Bush A, Hurst JR, Kotecha S, McGarvey L. Lung consequences in adults born prematurely. Postgrad Med J. 2015;91: 712-8.

8 Doyle LW, Adams AM, Robertson C, Ranganathan S, Davis NM, Lee KJ, et al. Increasing airway obstruction from 8 to 18 years in extremely preterm/low-birthweight survivors born in the surfactant era. Thorax. 2017; 72(8):712-9.
9 Islam JY, Keller RL, Aschner JL, Hartert TV, Moore PE. Understanding the short- and long-term respiratory outcomes of prematurity and bronchopulmonary dysplasia. Am J Respir Crit Care Med. 2015;192(2):134-56.

10 Karagianni P, Tsakalidis C, Kyriakidou M, Mitsiakos G, Chatziioanidis H, Porpodi M, et al. Neuromotor outcomes in infants with bronchopulmonary dysplasia. Pediatr Neurol. 2011 Jan;44(1):40-6.

11 Anderson PJ, Doyle LW. Neurodevelopmental outcome of bronchopulmonary dysplasia. Semin Perinatol. 2006;30(4):227-32.

12 Cheong JLY, Doyle LW. An update on pulmonary and neurodevelopmental outcomes of bronchopulmonary dysplasia. Semin Perinatol. 2018;42(7):478-84. 
13 Doyle LW, Carse E, Adams AM, Ranganathan S, Opie G, Cheong JLY. Ventilation in extremely preterm infants and respiratory function at 8 years. N Engl J Med. 2017; 377(4):329-37.

14 Roberts CL, Badgery-Parker T, Algert CS, Bowen JR, Nassar N. Trends in use of neonatal CPAP: a population-based study. BMC Pediatr. 2011;11:89.

15 Morley CJ, Davis PG, Doyle LW, Brion LP, Hascoet JM, Carlin JB. Nasal CPAP or intubation at birth for very preterm infants. $\mathrm{N}$ Engl J Med. 2008;358(7):700-8.

16 Hillman NH, Moss TJ, Nitsos I, Jobe AH. Moderate tidal volumes and oxygen exposure during initiation of ventilation in preterm fetal sheep. Pediatr Res. 2012;72(6):593-9.

17 Lines AL, Davey MG, Harding R, Hooper SB. Effect of increased lung expansion on surfactant protein mRNA levels in lambs. Pediatr Res. 2001;50(6):720-5.

18 Niemarkt HJ, Kuypers E, Jellema R, Ophelders D, Hütten M, Nikiforou M, et al. Effects of less-invasive surfactant administration on oxygenation, pulmonary surfactant distribution, and lung compliance in spontaneously breathing preterm lambs. Pediatr Res. 2014; 76(2):166-70.

19 Donnelly CG, Quinn CT, Nielsen SG, Raidal SL. Respiratory support for pharmacologically induced hypoxia in neonatal calves. Vet Med Int. 2016;2016:2129362-4.

20 Mayer CA, Martin RJ, Macfarlane PM. Increased airway reactivity in a neonatal mouse model of continuous positive airway pressure. Pediatr Res. 2015;78(2):145-51.

21 Reyburn B, Di Fiore JM, Raffay T, Martin RJ, Prakash YS, Jafri A, et al. The effect of continuous positive airway pressure in a mouse model of hyperoxic neonatal lung injury. Neonatology. 2016;109(1):6-13.

22 Gie AG, Salaets T, Vignero J, Regin Y, Vanoirbeek J, Deprest J, et al. Intermittent CPAP limits hyperoxia-induced lung damage in a rabbit model of bronchopulmonary dysplasia. Am J Physiol Lung Cell Mol Physiol. 2020; 318(5):L976-87.

23 Polglase GR, Hillman NH, Ball MK, Kramer BW, Kallapur SG, Jobe AH, et al. Lung and systemic inflammation in preterm lambs on continuous positive airway pressure or conventional ventilation. Pediatr Res. 2009;65(1): 67-71.

24 Kothe TB, Royse E, Kemp MW, Schmidt A, Salomone F, Saito M, et al. Effects of budesonide and surfactant in preterm fetal sheep. Am J Physiol Lung Cell Mol Physiol. 2018;315(2):L193-201.
25 Kothe TB, Royse E, Kemp MW, Usuda H, Saito M, Musk GC, et al. Epidermal growth factor receptor inhibition with Gefitinib does not alter lung responses to mechanical ventilation in fetal, preterm lambs. PLoS One. 2018;13(7): $\mathrm{e} 0200713$

26 Mulrooney N, Champion Z, Moss TJ, Nitsos I, Ikegami M, Jobe AH. Surfactant and physiologic responses of preterm lambs to continuous positive airway pressure. Am J Respir Crit Care Med. 2005;171(5):488-93.

27 Pillow JJ, Hillman N, Moss TJ, Polglase G, Bold G, Beaumont C, et al. Bubble continuous positive airway pressure enhances lung volume and gas exchange in preterm lambs. Am J Respir Crit Care Med. 2007;176(1):63-9.

28 Todd DA, John E, Osborn RA. Tracheal damage following conventional and high-frequency ventilation at low and high humidity. Crit Care Med. 1991;19(10):1310-6.

29 Nold JL, Meyers PA, Worwa CT, Goertz RH, Huseby K, Schauer G, et al. Decreased lung injury after surfactant in piglets treated with continuous positive airway pressure or synchronized intermittent mandatory ventilation. Neonatology. 2007;92(1):19-25.

30 Rey-Santano C, Mielgo VE, Gomez-Solaetxe MA, Ricci F, Bianco F, Salomone F, et al. Noninvasive ventilation and surfactant treatment as the primary mode of respiratory support in surfactant-deficient newborn piglets. Pediatr Res. 2018;83(4):904-14.

31 Dargaville PA, Lavizzari A, Padoin P, Black D, Zonneveld E, Perkins E, et al. An authentic animal model of the very preterm infant on nasal continuous positive airway pressure. Intensive Care Med Exp. 2015;3(1):51.

32 De Matteo R, Ishak N, Hanita T, Harding R, Sozo F. Respiratory adaptation and surfactant composition of unanesthetized male and female lambs differ for up to $8 \mathrm{~h}$ after preterm birth. Pediatr Res. 2016;79(1-1):13-21.

33 Thomson MA, Yoder BA, Winter VT, Martin $\mathrm{H}$, Catland D, Siler-Khodr TM, et al. Treatment of immature baboons for 28 days with early nasal continuous positive airway pressure. Am J Respir Crit Care Med. 2004;169(9): 1054-62.

34 Thomson MA, Yoder BA, Winter VT, Giavedoni L, Chang LY, Coalson JJ. Delayed extubation to nasal continuous positive airway pressure in the immature baboon model of bronchopulmonary dysplasia: lung clinical and pathological findings. Pediatrics. 2006; 118(5):2038-50.
35 Martin LD, Wetzel RC, Bilenki AL. Airway pressure release ventilation in a neonatal lamb model of acute lung injury. Crit Care Med. 1991;19(3):373-8.

36 Veneroni C, Dahl MJ, Lavizzari A, Dawson E, Rebentisch A, Studstill S, et al. Non-invasive measurements of respiratory system mechanical properties by the forced oscillation technique in spontaneously breathing, mixedbreed, normal term lambs from birth to five months of age. Physiol Meas. 2019;40(10): 105007.

37 Reyburn B, Li M, Metcalfe DB, Kroll NJ, Alvord J, Wint $A$, et al. Nasal ventilation alters mesenchymal cell turnover and improves alveolarization in preterm lambs. Am J Respir Crit Care Med. 2008;178(4):407-18.

38 Null DM, Alvord J, Leavitt W, Wint A, Dahl MJ, Presson AP, et al. High-frequency nasal ventilation for $21 \mathrm{~d}$ maintains gas exchange with lower respiratory pressures and promotes alveolarization in preterm lambs. Pediatr Res. 2014;75(4):507-16.

39 Thome UH, Genzel-Boroviczeny O, Bohnhorst B, Schmid M, Fuchs H, Rohde O, et al. Permissive hypercapnia in extremely low birthweight infants (PHELBI): a randomised controlled multicentre trial. Lancet Respir Med. 2015 Jul 1 [cited 2019 Dec 9]; 3(7):53443.

40 Lam R, Schilling D, Scottoline B, Platteau A, Niederhausen M, Lund KC, et al. The effect of extended continuous positive airway pressure on changes in lung volumes in stable premature infants: a randomized controlled trial. J Pediatr. 2020 Feb;217:66-72.e1.

41 Sandberg KL, Hjalmarson O. CPAP of $4 \mathrm{~cm}$ $\mathrm{H} 2 \mathrm{O}$ has no short-term benefit at term in infants with BPD. Neonatology. 2012;102(4): 282-6.

42 Hütten MC, Kuypers E, Ophelders DR, Nikiforou M, Jellema RK, Niemarkt HJ, et al. Nebulization of Poractant alfa via a vibrating membrane nebulizer in spontaneously breathing preterm lambs with binasal continuous positive pressure ventilation. Pediatr Res. 2015;78(6):664-9.

43 Yeh TF, Chen CM, Wu SY, Husan Z, Li TC, Hsieh WS, et al. Intratracheal administration of budesonide/surfactant to prevent bronchopulmonary dysplasia. Am J Respir Crit Care Med. 2016;193(1):86-95.

44 Kothe TB, Sadiq FH, Burleyson N, Williams HL, Anderson C, Hillman NH. Surfactant and budesonide for respiratory distress syndrome: an observational study. Pediatr Res. 2020 Apr;87(5):940-5. 